\title{
ALAIN GRIGIS
}

FRÉDÉRIC KLOPP

\section{Valeurs propres et résonances au voisinage d'un seuil}

Bulletin de la S. M. F., tome 124, no 3 (1996), p. 477-501

<http://www.numdam.org/item?id=BSMF_1996_124_3_477_0>

C B Bulletin de la S. M. F., 1996, tous droits réservés.

L'accès aux archives de la revue «Bulletin de la S. M. F. » (http: //smf.emath.fr/Publications/Bulletin/Presentation.html) implique l'accord avec les conditions générales d'utilisation (http://www.numdam.org/ conditions). Toute utilisation commerciale ou impression systématique est constitutive d'une infraction pénale. Toute copie ou impression de ce fichier doit contenir la présente mention de copyright.

\section{Numdam}


Bull. Soc. math. France,

124,1996 , p. $477-501$.

\title{
VALEURS PROPRES ET RÉSONANCES AU VOISINAGE D'UN SEUIL
}

\author{
PAR \\ ALAIN GRIGIS Et FRÉDÉRIC KLOPP $\left({ }^{*}\right)$
}

RÉSUMÉ. - Nous étudions dans un cadre abstrait un opérateur dépendant d'un paramètre dont la résolvante peut s'étendre de façon méromorphe à une surface de Riemann au voisinage d'un point de branchement. Nous étudions la dépendance des pôles de la résolvante, ou résonances en fonction du paramètre; en particulier, nous étudions le cas où une résonance se trouve au point de branchement. Cette étude peut s'appliquer à un opérateur de Schrödinger à potentiel périodique avec une perturbation compacte, le paramètre étant une constante de couplage, et le point de branchement ou seuil, l'extrémité d'une bande de spectre absolument continu.

ABSTRACT. - In an abstract setting, we consider an operator whose resolvent can be extended meromorphically to a Riemann surface near a branching point. The operator depends on a parameter, and we study the behavior of the resonances i.e. poles of the resolvent as functions of the parameter; in particular, we study what happens when a resonance is located at the branching point. This study may be applied to a periodic Schrödinger operator perturbed by a compactly supported potential. The parameter is a coupling constant and the branching point is an edge of the absolutely continuous spectrum of the periodic operator.

\section{Introduction}

On considère sur $\mathbb{R}^{n}$ un opérateur de Schrödinger avec un potentiel périodique

$$
H=-\Delta+V
$$

${ }^{*}$ ) Texte recu le 28 juin 1995, accepté le 31 juillet 1995.

A. Grigis, Département de Maths, Institut Galilée, Université Paris-Nord, av. JeanBaptiste Clément, 93430 Villetaneuse (France).

Email : grigis@math.univ-paris13.fr.

F. KLOPP, Département de Mathématique, Bât. 425, Université de Paris-Sud, Centre d'Orsay, 91405 Orsay CEDEx (France) et Department of Mathematics, The Johns Hopkins University, 3400 N. Charles St., Baltimore, 21218 MD (U.S.A).

Email : klopp@lanors.matups.fr.

Classification AMS : 35 P 25, 35 P 20, 35 J 10, 47 A 40, 81 H 20. 
où $V$ est $\Gamma$-périodique, $\Gamma$ étant un réseau de $\mathbb{R}^{n}$. Il est bien connu que le spectre de cet opérateur est purement absolument continu et possède une structure de bandes. La résolvante $(\lambda-H)^{-1}$ peut se prolonger à partir de la région physique $\{\lambda \in \mathbb{C} ; \operatorname{Im} \lambda>0\}$ au demi-plan inférieur à travers les bandes spectrales, comme cela a été démontré par C. Gérard [Gé2]. On peut prolonger cette résolvante à une surface de Riemann qui présente des points de branchements (en particulier aux extrémités des bandes spectrales de $H$ ) appelés seuils.

Soit $W$ un potentiel à support compact et positif. Pour $t$ une constante de couplage réelle, on définit l'opérateur perturbé

$$
H(t)=H+t W=-\Delta+V+t W .
$$

Celui-ci peut admettre des valeurs propres dans les lacunes du spectre continu. On peut également construire un prolongement méromorphe de la résolvante de $H(t)$ à la surface de Riemann citée ci-dessus. Sur cette surface de Riemann, $H(t)$ peut admettre des résonances. Dans [Kl], le second auteur a étudié, dans la limite semi-classique, la création de telles valeurs propres et résonances pour une petite constante de couplage.

De nombreux travaux, par exemple [ADH], [Bi], [Rai], [Sob] étudient l'asymptotique du nombre de valeurs propres de $H(t)$ dans les lacunes du spectre continu pour les grandes valeurs de $t$. Comme $W$ est supposé positif, il est clair que ces valeurs propres sont des fonctions croissantes de $t$. $\mathrm{Au}$ cours d'un colloque organisé à l'Institut Euler à Saint-Pétersbourg, M.Sh. Birman a posé la question suivante :

«Que deviennent les valeurs propres lorsque l'on fait varier la constante de couplage? On sait qu'elles croissent avec celle-ci jusqu'à rencontrer la prochaine bande de spectre absolument continu. Mais là que se passe-t-il? Deviennent-elles des résonances ou des valeurs propres plongées?»

C'est cette question qui a motivé la présente étude. Nous avons donc repris dans un cadre abstrait la méthode d'extension de la résolvante à une surface de Riemann, pour un opérateur non perturbé tout d'abord. Ensuite, nous introduisons une perturbation compacte dépendant analytiquement d'un paramètre $t$. Nous définissons les résonances et nous étudions leur dépendance par rapport au paramètre $t$. La situation vraiment nouvelle est celle où pour une valeur $t_{0}$ de la constante de couplage, une résonance est située au seuil i.e au point de ramification de la surface de Riemann. Le comportement de la résonance lorsque $t$ varie dépend alors de façon essentielle du type de ramification du seuil. Sous des hypothèses génériques, nous obtenons l'un des trois cas suivants :

1) Il existe une unique résonance $z(t)$ telle que $z\left(t_{0}\right)=z_{0}, z_{0}$ étant le seuil en question.

TOME $124-1996-\mathrm{N}^{\circ} 3$ 
Dans le cas d'une perturbation d'un opérateur de Schrödinger périodique, c'est ce qui se passe en dimension 3 . Ainsi la réponse à la question est que la valeur propre arrive au seuil, se transforme en résonance et repart.

2) Il existe un nombre fini de résonances $\left(z_{i}\right)_{1 \leq i \leq q}$ telles que $z_{i}\left(t_{0}\right)=z_{0}$. pour $1 \leq i \leq q$.

Dans le cas d'une perturbation d'un opérateur de Schrödinger périodique, c'est ce qui se passe en dimension impaire supérieure à 3 . Dans ce cas, $q=2$. La surface de Riemann a deux feuillets. Le feuillet physique contient au voisinage du seuil uniquement la valeur propre $z_{1}(t), t>t_{0}$, plus précisément le relevé de la valeur propre. L'autre feuillet contient la résonance $z_{2}(t), t>t_{0}$, et les 2 résonances $z_{1}(t)$ et $z_{2}(t)$ pour $t<t_{0}$. Ces branches de résonances sont données par une équation implicite de la forme

$$
a_{1,0,0} t+a_{0,1,0} z^{2}+a_{0,0,1} z^{n-2}+\sum_{\substack{j+k+\ell \geq 2 \\ j, k, \ell \geq 0}} a_{j, k, \ell} t^{j} z^{2 k+(n-2) \ell}=0 .
$$

Nous discutons la non-annulation des coefficients principaux.

Le fait que les résonances arrivent par paires provient uniquement du fait que la ramification de la surface de Riemann dans ce cas est du type rationnel quadratique (voir la discussion suivant le théorème 2.1). Il n'y a pas de raison que les projections de ces deux branches sur la base, où vit le paramètre spectral, soient confondues. L'existence des deux familles de résonances n'est pas dû au choix de l'uniformisation de la variable $t$ ou $\mathrm{du}$ paramètre spectral.

3) Il existe une infinité dénombrable de résonances $\left(z_{i}\right)_{i \in \mathbb{N}}$ telles que $z_{i}\left(t_{0}\right)=z_{0}$ pour $i \in \mathbb{N}$.

Dans le cas d'une perturbation d'un opérateur de Schrödinger périodique, c'est ce qui se passe en dimension paire supérieure à 3 . Le nombre infini de résonances provient du fait que dans ce cas-là, la ramification de la surface de Riemann est du type logarithmique. Mis à part le fait qu'elles sont plus nombreuses, les résonances se comportent de la même façon que dans le cas 2 .

Une partie des hypothèses que nous faisons sont génériques au sens mathématique. Cela dit, dans l'application au cas d'un opérateur de Schrödinger périodique perturbé, nous n'avons pas cherché sous quelles conditions portant sur le potentiel périodique et sur la perturbation les dites hypothèses sont vérifiées. Il est probable qu'elles le soient en général 
mais c'est une question que nous n'avons pas examinée. D'autre part, nous n'avons pas non plus cherché à trouver une interprétation physique aux résonances qui accompagnent la valeur propre. Ces questions ont peut-être un intérêt.

Le plan de l'article est le suivant. Dans la première partie, nous considérons un opérateur dont la résolvante admet un prolongement holomorphe ramifié puis nous démontrons, dans un cadre abstrait, pour un tel opérateur perturbé, l'existence d'un prolongement méromorphe au voisinage d'un point de ramification ou seuil. Ce résultat est local. Nous définissons les résonances. Dans la deuxième partie, nous étudions l'éclatement d'une résonance située à un seuil suivant le type de ramification de la surface de Riemann à ce seuil.

Remerciements. - Les auteurs remercient le Professeur Buslaev pour son accueil à l'Institut Euler en septembre 1993.

\section{Prolongement méromorphe de la résolvante}

\subsection{L'opérateur non perturbé.}

Soit $\mathcal{H}_{0}$ un espace de Hilbert (on note $\langle\cdot, \cdot\rangle_{0}$ son produit scalaire) et $H$ un opérateur auto-adjoint sur $\mathcal{H}_{0}$ de domaine $D(H)$ dense dans $\mathcal{H}_{0}$. On notera $\mathcal{H}_{1}=D(H)$, le domaine de $H$ muni du produit scalaire du graphe

$$
\langle\cdot, \cdot\rangle_{1}=\langle\cdot, \cdot\rangle_{0}+\langle H \cdot, H \cdot\rangle_{0}=\langle(H+i) \cdot,(H+i) \cdot\rangle_{0} .
$$

Comme $H$ est auto-adjoint donc fermé, $\mathcal{H}_{1}$ est un espace de Hilbert. L'opérateur $H$ est borné de $\mathcal{H}_{1}$ dans $\mathcal{H}_{0}$, et pour $z \in \mathbb{C} \backslash \mathbb{R}$, l'opérateur $(z-H)^{-1}$ est borné de $\mathcal{H}_{0}$ dans $\mathcal{H}_{1}$. On suppose qu'il existe deux espaces de Hilbert $\mathcal{A}_{0}$ et $\mathcal{A}_{1}$, tels que

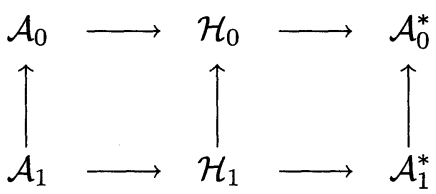

les inclusions étant toutes continues et denses (ici $\mathcal{A}_{0}^{*}$ (resp. $\mathcal{A}_{1}^{*}$ ) est le dual de $\mathcal{A}_{0}$ (resp. $\left.\mathcal{A}_{1}\right)$ pour $\langle\cdot, \cdot\rangle_{0}$ (resp. $\langle\cdot, \cdot\rangle_{1}$ ). On suppose de plus que

$$
(H+i)\left(\mathcal{A}_{1}\right) \subset \mathcal{A}_{0} \quad \text { et } \quad(H+i)^{-1}\left(\mathcal{A}_{0}\right) \subset \mathcal{A}_{1} .
$$

Le théorème du graphe fermé nous dit alors que $H+i$ est une bijection bicontinue de $\mathcal{A}_{1}$ dans $\mathcal{A}_{0}$. On prolonge $H+i$ en un opérateur bijectif bicontinu de $\mathcal{A}_{1}^{*}$ dans $\mathcal{A}_{0}^{*}$.

TOME $124-1996-\mathrm{N}^{\circ} 3$ 


\subsection{Hypothèses sur la résolvante au bord d'une lacune spec- trale.}

On note $\sigma(H)$ le spectre de $H$. On va supposer que le spectre de $H$ évite un intervalle ] $a, a+\epsilon_{0}$ [ et que la résolvante de $H$ peut se prolonger près de $a$ en tant qu'opérateur borné sur un ensemble de vecteurs analytiques. Plus précisément, après s'être ramené à $a=0$, on fait l'hypothèse :

Hypothèse (H.1).

a) Il existe $\epsilon_{0}>0, S$, une fonction analytique $\left.\left.\operatorname{sur} D_{\mathbb{C}}\left(0, \epsilon_{0}\right) \backslash\right]-\epsilon_{0}, 0\right]$, $F$ et $G$ des fonctions analytiques non nulles de $D_{\mathbb{C}}\left(0, \epsilon_{0}\right)$ à valeurs dans $\mathcal{B}\left(\mathcal{A}_{0}, \mathcal{A}_{1}^{*}\right)$ telles que, pour $\left.\left.\lambda \in D_{\mathbb{C}}\left(0, \epsilon_{0}\right) \backslash\right]-\epsilon_{0}, 0\right]$,

$$
(\lambda-H)^{-1}=F(\lambda)+S(\lambda) \cdot G(\lambda) .
$$

b) $S$ admet un prolongement analytique $\widetilde{S}$ à un revêtement analytique $\mathcal{R}_{\epsilon_{0}} d u$ disque pointé $D_{\mathbb{C}}\left(0, \epsilon_{0}\right) \backslash\{0\}$; on note $\rho$, la projection canonique de $\mathcal{R}_{\epsilon_{0}}$ sur $D_{\mathbb{C}}\left(0, \epsilon_{0}\right) \backslash\{0\}$;

c) $S(\lambda)$ tend vers 0 quand $\lambda$ tend vers 0 et $\left.\left.\lambda \in D_{\mathbb{C}}\left(0, \epsilon_{0}\right) \backslash\right]-\epsilon_{0}, 0\right]$ et, pour $\lambda \in] 0, \epsilon_{0}[, S(\lambda)$ appartient à $\mathbb{R}$.

\section{REMARQues.}

1) L'hypothèse (H.1) a) permet de dire que $H$ n'a pas de spectre dans ] $0, \epsilon_{0}$ [ (en utilisant la formule de Stone ( $c f$. [ReSi]) et le fait que $\mathcal{A}_{1}$ est dense dans $\mathcal{H}_{0}$ ). De plus, comme $S$ se prolonge analytiquement à ] $-\epsilon_{0}, 0[$, le principe d'absorption limite dit que le spectre de $H$ dans ] $-\epsilon_{0}, 0$ ] est purement absolument continu ( $c f$. [CFKS, chap. 4]).

2) On suppose que $S$ ne se prolonge pas analytiquement à un voisinage de 0 ; sinon 0 et donc un voisinage de 0 ne seraient pas dans le spectre de $H$, ce qui est un cas sans intérêt.

On suppose donc que 0 est un seuil, c'est-à-dire un point de branchement pour $S(\lambda)$. Plus tard, on rajoutera un point à $\mathcal{R}_{\epsilon_{0}}$ pour définir la fibre au-dessus de 0 . Ceci sera fait dans la partie 1.6 et précisé dans la partie 2 .

3) Si (H.1) est vérifiée, on peut prolonger la résolvante de $H$ à $\mathcal{R}_{\epsilon_{0}}$ en tant qu'opérateur borné de $\mathcal{A}_{0}$ dans $\mathcal{A}_{1}^{*}$ de la façon suivante : pour $z \in \mathcal{R}_{\epsilon_{0}}$, on définit

$$
R_{H}(z)=F(\rho(z))+\widetilde{S}(z) \cdot G(\rho(z)) .
$$

4) Comme $S(\lambda)$ est réelle pour $\lambda$ réel, on a, pour $\left.\left.\lambda \in D_{\mathbb{C}}\left(0, \epsilon_{0}\right) \backslash\right]-\epsilon_{0}, 0\right]$, $\overline{S(\bar{\lambda})}=S(\lambda)$. De plus $H$ est auto-adjoint, ainsi pour $\phi \in \mathcal{A}_{0}$ et $\psi \in \mathcal{A}_{0}$, 
on sait que $\left\langle(\lambda-H)^{-1} \phi, \psi\right\rangle_{0}=\left\langle\phi,(\bar{\lambda}-H)^{-1} \psi\right\rangle_{0}$, c'est-à-dire

$$
\left\langle F^{*}(\bar{\lambda}) \phi, \psi\right\rangle_{0}+\overline{S(\bar{\lambda})}\left\langle G^{*}(\bar{\lambda}) \phi, \psi\right\rangle_{0}=\langle F(\lambda) \phi, \psi\rangle_{0}+S(\lambda)\langle G(\lambda) \phi, \psi\rangle_{0}
$$

donc

$$
\left\langle F^{*}(\bar{\lambda}) \phi, \psi\right\rangle_{0}-\langle F(\lambda) \phi, \psi\rangle_{0}=S(\lambda)\left(\langle G(\lambda) \phi, \psi\rangle_{0}-\left\langle G^{*}(\bar{\lambda}) \phi, \psi\right\rangle_{0}\right) .
$$

Comme $\langle F(\lambda) \phi, \psi\rangle_{0},\langle G(\lambda) \phi, \psi\rangle_{0},\left\langle F^{*}(\bar{\lambda}) \phi, \psi\right\rangle_{0}$ et $\left\langle G^{*}(\bar{\lambda}) \phi, \psi\right\rangle_{0}$ sont analytiques en $\lambda$, on en déduit que, pour $\lambda$ réel, $F(\lambda)$ et $G(\lambda)$ sont symétriques (i.e. $\langle F(\lambda) \phi, \psi\rangle_{0}=\langle\phi, F(\lambda) \psi\rangle_{0}$ et $\langle G(\lambda) \phi, \psi\rangle_{0}=\langle\phi, G(\lambda) \psi\rangle_{0}$ pour $\lambda$ réel, $\phi \in \mathcal{A}_{0}$ et $\left.\psi \in \mathcal{A}_{0}\right)$

Cas de l'opérateur de Schrödinger.

L'hypothèse (H.1) est vérifiée en dimension supérieure ou égale à 3 , pour le laplacien libre $(c f .[\mathrm{BaSk}])$ ainsi que pour des opérateurs de Schrödinger périodiques ( $c f$. [Gé2], [Gé1]). Dans ce cas, les espaces $\mathcal{A}_{0}$ et $\mathcal{A}_{1}^{*}$ sont

$$
\begin{aligned}
& \mathcal{A}_{0}=L_{a}^{2}\left(\mathbb{R}^{n}\right)=\left\{u \in L_{\mathrm{loc}}^{2}\left(\mathbb{R}^{n}\right) ; \mathrm{e}^{a\langle x\rangle} u \in L^{2}\left(\mathbb{R}^{n}\right)\right\}, \\
& \mathcal{A}_{1}^{*}=H_{-a}^{2}\left(\mathbb{R}^{n}\right)=\left\{u \in H_{\mathrm{loc}}^{2}\left(\mathbb{R}^{n}\right) ; \mathrm{e}^{-a\langle x\rangle} u \in L^{2}\left(\mathbb{R}^{n}\right)\right\},
\end{aligned}
$$

où $\langle x\rangle=\sqrt{1+x^{2}}$ et $a>0$. Ces espaces sont équipés de leur norme naturelle.

De plus, dans le cas des opérateurs de Schrödinger périodiques, si l'extrémité de la bande spectrale considérée est simple, on a

$$
S(\lambda)= \begin{cases}\lambda^{(n-2) / 2} & \text { en dimension } n \text { impaire } \\ \lambda^{(n-2) / 2} \log \lambda & \text { en dimension } n \text { paire (type logarithmique). }\end{cases}
$$

Remarquons que l'hypothèse (H.1) c) est alors vérifiée si la dimension $n$ est $\geq 3$. On pourrait choisir $S(\lambda)=\lambda^{\frac{1}{2}}$ ou $S(\lambda)=\lambda \log \lambda$ et incorporer les facteurs réguliers dans $G(\lambda)$. Évidemment, cela ne changerait rien aux résultats.

\subsection{Décomposition spectrale.}

Notons :

- $\Pi_{+}$la projection spectrale associée à $H$ sur $\left[\frac{1}{2} \epsilon_{0},+\infty[\right.$ et

- $\Pi_{-}=\mathbf{1}-\Pi_{+}$, celle sur $\left.]-\infty, \frac{1}{2} \epsilon_{0}\right]$.

On suppose que : 
Hypothèse $($ H.2 $) \quad \Pi_{+}\left(\mathcal{A}_{0}\right) \subset \mathcal{A}_{0}$.

En utilisant le fait que $\Pi_{+}$est borné sur $\mathcal{H}_{0}$ et le théorème du graphe fermé, on voit que $\Pi_{+}$est borné sur $\mathcal{A}_{0}$. De plus, on peut étendre $\Pi_{+}$en un opérateur borné sur $\mathcal{A}_{0}^{*}$ par, pour $\psi^{*} \in \mathcal{A}_{0}^{*}$,

$$
\forall \psi \in \mathcal{A}_{0}, \quad\left\langle\Pi_{+} \psi^{*}, \psi\right\rangle=\left\langle\psi^{*}, \Pi_{+} \psi\right\rangle
$$

On voit que $\Pi_{+}$laisse $\mathcal{H}_{1}$ et $\mathcal{A}_{1}$ invariant en utilisant l'identité

$$
\Pi_{+}(H+i)^{-1}=(H+i)^{-1} \Pi_{+}
$$

vraie sur $\mathcal{H}_{0}$. Ainsi $\Pi_{+}$est borné sur $\mathcal{H}_{1}$ et sur $\mathcal{A}_{1}$, et s'étend en un opérateur borné sur $\mathcal{A}_{1}^{*}$. Tout ceci reste vrai pour $\Pi_{-}$.

\subsection{Hypothèses sur la perturbation.}

On va maintenant définir la perturbation de $H$. Soit

$$
(A(t))_{t \in D_{\mathbb{C}}\left(0, \epsilon_{0}\right)}
$$

une famille analytique en $t$ d'opérateurs sur $\mathcal{H}_{0}$ de domaine $D(A) \supset D(H)$ indépendant de $t$, telle que :

Hypothèse (H.3).

a) pour $t \in D_{\mathbb{C}}\left(0, \epsilon_{0}\right), A(t)$ est compact de $\mathcal{A}_{1}^{*}$ dans $\mathcal{A}_{0}$ pour $t$ appartenant $\grave{a}]-\epsilon_{0}, \epsilon_{0}[$;

b) pour $t \in]-\epsilon_{0}, \epsilon_{0}[, A(t)$ est autoadjoint;

c) $1-\Pi_{-} A(0) \Pi_{-} F(0)$ est inversible en tant qu'opérateur de $\mathcal{A}_{0}$ dans lui-même.

REMARQUes.

1) Le point a) est équivalent à supposer que $A$ est $H$-compact de $\mathcal{A}_{0}^{*}$ dans $\mathcal{A}_{0}$. Ceci implique que $A$ est $H$-compact sur $\mathcal{H}_{0}$.

2) Par le point a), on sait que $\Pi_{-} A(0) \Pi_{-} F(0)$ est compact de $\mathcal{A}_{0}$ dans lui-même. Donc supposer c) revient à supposer que 1 n'est pas valeur propre de $\Pi_{-} A(0) \Pi_{-} F(0)$. Comme $F(0) \Pi_{-} A(0) \Pi_{-}$est adjoint de $\Pi_{-} A(0) \Pi_{-} F(0)$, on peux supposer de façon équivalente que

$$
1-F(0) \Pi_{-} A(0) \Pi_{-}
$$

est inversible en tant qu'opérateur de $\mathcal{A}_{1}^{*}$ dans lui-même. 


\subsection{L'opérateur de Livsic.}

Sous l'hypothèse (H.3) ( $c f$. [ReSi]), pour $t$ réel,

$$
H(t)=H+A(t)
$$

est un opérateur auto-adjoint de domaine $D(H)$, et le spectre de $H(t)$ dans $] 0, \epsilon_{0}[$ est discret.

Soient $\varphi \in D(H), \psi \in \mathcal{H}_{0}$ et $\left.\lambda \in\right] 0, \epsilon_{0}[$. On note :

$$
\varphi_{+}=\Pi_{+} \varphi, \quad \varphi_{-}=\Pi_{-} \varphi \text {. }
$$

Alors :

$$
\begin{aligned}
(H(t)-\lambda) \varphi & =\psi \\
& \Longleftrightarrow\left\{\begin{array}{l}
\left(H_{+}+A_{++}(t)-\lambda\right) \varphi_{+}+A_{+-}(t) \varphi_{-}=\psi_{+}, \\
\left(H_{-}+A_{--}(t)-\lambda\right) \varphi_{-}+A_{-+}(t) \varphi_{+}=\psi_{-},
\end{array}\right.
\end{aligned}
$$

où, avec $\epsilon, \epsilon^{\prime} \in\{+,-\}$ :

$$
\begin{gathered}
H_{\epsilon}=\Pi_{\epsilon} H \Pi_{\epsilon}=\Pi_{\epsilon} H=H \Pi_{\epsilon}, \\
A_{\epsilon \epsilon^{\prime}}(t)=\Pi_{\epsilon} A(t) \Pi_{\epsilon^{\prime}},
\end{gathered}
$$

Pour $\lambda>0, \Pi_{-}\left(\lambda-H_{-}\right)^{-1} \Pi_{-}$est borné de $\mathcal{H}_{0}$ dans $\mathcal{H}_{1}$. Ainsi d'après les hypothèses (H.1) a) et c), et (H.3) c), pour $\lambda>0$ suffisamment proche de 0 et $t$ assez petit, $1-\left(\lambda-H_{-}\right)^{-1} A_{--}(0)$ est inversible de $\mathcal{A}_{1}^{*}$ dans lui-même, donc de $\mathcal{H}_{1}$ dans $\mathcal{H}_{1}$. Donc

$$
\Pi_{-}\left(\lambda-H_{-}-A_{--}(t)\right)^{-1} \Pi_{-}=\left(1-\left(\lambda-H_{-}\right)^{-1} A_{--}(t)\right)^{-1} \Pi_{-}\left(\lambda-H_{-}\right)^{-1} \Pi_{-}
$$

est borné de $\mathcal{H}_{0}$ dans $\mathcal{H}_{1}$. Ainsi (1.1) devient

$$
\left\{\begin{aligned}
\Pi_{-}\left(\lambda-H_{-}-A_{--}(t)\right)^{-1} \cdot A_{-+}(t) \varphi_{+} & \\
& -\left(\lambda-H_{-}-A_{--}(t)\right)^{-1} \psi_{-}=\varphi_{-}, \\
\left(L_{+}(t, \lambda)-\lambda\right) \varphi_{+}= & \psi_{+}+A_{+-}(t)\left(\lambda-H_{-}-A_{--}(t)\right)^{-1} \psi_{-},
\end{aligned}\right.
$$

où $L_{+}(t, \lambda)$ est l'opérateur de Livsic ( $c f$. [Or], [Ho]) défini par :

$$
L_{+}(t, \lambda)=H_{+}+A_{++}(t)+A_{+-}(t)\left(\lambda-H_{-}-A_{--}(t)\right)^{-1} A_{-+}(t) .
$$

Pour $\lambda \in] 0, \epsilon_{0}[$, on définit :

$$
R_{-}(t, \lambda)=\Pi_{-}\left(\lambda-H_{-}-A_{--}(t)\right)^{-1} \Pi_{-} .
$$

On a la : 
Proposition 1.1. - Sous les hypothèses (H.1)-(H.3), il existe $\epsilon>0$, $\Gamma_{+}(t, \lambda, z)$, une fonction analytique de $B_{\mathbb{C}^{3}}(0, \epsilon)$ à valeurs dans les opérateurs $H_{+}$-compacts sur $\mathcal{H}_{0}$ et $R_{-}(t, \lambda, z)$, une fonction analytique de $B_{\mathbb{C}^{3}}(0, \epsilon)$ à valeurs dans les opérateurs bornés de $\mathcal{A}_{0}$ dans $\mathcal{A}_{1}^{*}$ telles que, si $\left.\lambda \in D_{\mathbb{C}}\left(0, \epsilon_{0}\right) \backslash\right]-\epsilon_{0}, 0\left[\right.$ et $S(\lambda) \in B_{\mathbb{C}}(0, \epsilon)$, alors :

$$
\begin{gathered}
\Gamma_{+}(t, \lambda, S(\lambda))+H_{+}=L_{+}(t, \lambda), \\
R_{-}(t, \lambda, S(\lambda))=R_{-}(t, \lambda) .
\end{gathered}
$$

Si $z, t$ et $\lambda$ sont tous trois réels, l'opérateur $\Gamma_{+}(t, \lambda, z)$ est autoadjoint sur $\mathcal{H}$ et l'opérateur $R_{-}(t, \lambda, z)$ est symétrique sur $\mathcal{A}_{0}$ (i.e. $\left\langle R_{-}(t, \lambda, z) \phi, \psi\right\rangle=\left\langle\phi, R_{-}(t, \lambda, z) \psi\right\rangle$ pour $\phi$ et $\psi$ dans $\left.\mathcal{A}_{0}\right)$.

Preuve de la Proposition 1.1. - Pour $\lambda \notin \sigma(H)$, on écrit :

$$
\Pi_{-}\left(\lambda-H_{-}\right)^{-1} \Pi_{-}=(\lambda-H)^{-1}-\Pi_{+}\left(\lambda-H_{+}\right)^{-1} \Pi_{+} .
$$

Or $\Pi_{+}\left(\lambda-H_{+}\right)^{-1} \Pi_{+}$est analytique borné de $\mathcal{H}_{0}$ dans $\mathcal{H}_{1}$, donc de $\mathcal{A}_{0}$ dans $\mathcal{A}_{1}^{*}$, pour $\lambda$ dans un voisinage de 0 (comme le spectre de $H_{+}$ne contient pas ce voisinage de 0 ) donc, par l'hypothèse (H.1),

$$
\Pi_{-}\left(\lambda-H_{-}\right)^{-1} \Pi_{-}=F_{-}(\lambda)+S(\lambda) \cdot G_{-}(\lambda)
$$

où $F_{-}$et $G_{-}$ont les mêmes propriétés que $F$ et $G$ (car $H_{+}$est autoadjoint).

Pour $\lambda>0$ et $\lambda$ voisin de 0 , on écrit, en prenant soin de conserver la symétrie des expressions,

$$
\begin{aligned}
& R_{-}(t, \lambda)=\Pi_{-}\left(\lambda-H_{-}-A_{--}(t)\right)^{-1} \Pi_{-} \\
&=\frac{1}{2}\left\{\begin{array}{l}
\Pi_{-}\left(\lambda-H_{-}\right)^{-1} \Pi_{-}\left(1-A_{--}(t)\left(\lambda-H_{-}\right)^{-1} \Pi_{-}\right)^{-1} \Pi_{-} \\
+\Pi_{-}\left(1-\Pi_{-}\left(\lambda-H_{-}\right)^{-1} A_{--}(t)\right)^{-1} \\
\left.\times \Pi_{-}\left(\lambda-H_{-}\right)^{-1} \Pi_{-}\right\}
\end{array}\right. \\
&=\frac{1}{2}\left\{\begin{array}{r}
\left(F_{-}(\lambda)+S(\lambda) \cdot G_{-}(\lambda)\right) \\
\times\left(1-A_{--}(t) F_{-}(\lambda)-S(\lambda) A_{--}(t) G_{-}(\lambda)\right)^{-1} \Pi_{-} \\
+\Pi_{-}\left(1-F_{-}(\lambda) A_{--}(t)-S(\lambda) G_{-}(\lambda) A_{--}(t)\right)^{-1} \\
\left.\left(F_{-}(\lambda)+S(\lambda) \cdot G_{-}(\lambda)\right)\right\}
\end{array}\right.
\end{aligned}
$$


Pour $(t, \lambda, u) \in B_{\mathbb{C}^{3}}(0, \epsilon)$, définissons les opérateurs

$$
\begin{aligned}
R_{-}(t, \lambda, u)=\frac{1}{2}\{ & \left(F_{-}(\lambda)+u \cdot G_{-}(\lambda)\right) \\
& \times\left(1-A_{--}(t) F_{-}(\lambda)-u \cdot A_{--}(t) G_{-}(\lambda)\right)^{-1} \Pi_{-} \\
+ & \Pi_{-}\left(1-F_{-}(\lambda) A_{--}(t)-u \cdot G_{-}(\lambda) A_{--}(t)\right)^{-1} \\
& \left.\times\left(F_{-}(\lambda)+u \cdot G_{-}(\lambda)\right)\right\}
\end{aligned}
$$

On vérifie aisément que, pour $(t, \lambda)$ tels que $\left.\left.\lambda \in D_{\mathbb{C}}\left(0, \epsilon_{0}\right) \backslash\right]-\epsilon_{0}, 0\right]$ et $(t, S(\lambda)) \in B_{\mathbb{C}^{2}}(0, \epsilon)$, on a :

$$
R_{-}(t, \lambda, S(\lambda))=R_{-}(t, \lambda) \quad \text { et } \quad L_{+}(t, \lambda)=H_{+}+\Gamma_{+}(t, \lambda, S(\lambda)) .
$$

Par ce qui a été dit ci-dessus, il est clair que $R_{-}(t, \lambda, u)$ est borné de $\mathcal{A}_{0}$ dans $\mathcal{A}_{1}^{*}$. Pour $t, \lambda$ et $u$ réels, la symétrie de $R_{-}(t, \lambda, u)$ découle immédiatement de celles de $A(t), F$ et $G$ et de la symétrie imposée dans la définition de $R_{-}(t, \lambda, u)$. Ceci entraîne alors que $\Gamma_{+}(t, \lambda, u)$ est symétrique.

Comme $A(t)$ est compact de $\mathcal{A}_{1}^{*}$ dans $\mathcal{A}_{0}$, il est en particulier $H$ compact sur $\mathcal{H}_{0}$. Donc $A_{++}(t)$ est $H_{+}$-compact. Pour $\lambda$ voisin de 0 , $\left(\lambda-H_{+}\right)^{-1}$ est borné dans $\mathcal{H}_{0}$ et $A_{-+}(t)\left(\lambda-H_{+}\right)^{-1}$ est compact. De plus, $A_{+-}(t)$ est compact de $\mathcal{A}_{1}^{*}$ dans $\mathcal{A}_{0}$ et $R_{-}(t, \lambda, u)$ est borné de $\mathcal{A}_{0}$ dans $\mathcal{A}_{1}^{*}$, donc $\Gamma_{+}(t, \lambda, u)$ est $H_{+}$-compact. Ce qui implique que $\Gamma_{+}(t, \lambda, u)$ est auto-adjoint pour $t, \lambda$ et $u$ réels.

Équation aux valeurs propres.

On sait que le spectre de $H_{+}$est contenu dans $\left[\epsilon_{0},+\infty[;\right.$ ainsi, au voisinage de 0 , le spectre de $H_{+}+\Gamma_{+}(t, \lambda, u)$ est discret. Soient $\left(\mu_{j}(t, \lambda, u)\right)_{1 \leq j \leq m}$, les valeurs propres de $H_{+}+\Gamma_{+}(t, \lambda, u)$ dans $D\left(0, \epsilon_{0}\right)$. Comme $\Gamma_{+}(t, \lambda, u)$ est analytique en $(t, \lambda, u)$ et $H_{+}-$compact, on peut appliquer la théorie des perturbations analytiques à $H_{+}+\Gamma_{+}(t, \lambda, u)$. Entre autres, si $\mu_{0}$ est une valeur propre de $H_{+}+\Gamma_{+}\left(t_{0}, \lambda_{0}, u_{0}\right)$, alors il existe une application analytique $(t, \lambda, u, \mu) \mapsto k(t, \lambda, u, \mu)$, définie dans un voisinage $W$ de $\left(t_{0}, \lambda_{0}, u_{0}, \mu_{0}\right)$ telle que

(i) $k\left(t_{0}, \lambda_{0}, u_{0}, \mu_{0}\right)=0$,

(ii) pour $(t, \lambda, u, \mu) \in W, \mu$ est valeur propre de $H_{+}+\Gamma_{+}(t, \lambda, u)$ si et seulement si $k(t, \lambda, u, \mu)=0$.

Si $\mu_{0}$ est une valeur propre simple de $H_{+}+\Gamma_{+}(t, \lambda, u)$, alors on peut choisir

$$
k(t, \lambda, u, \mu)=\mu-\mu(t, \lambda, u)
$$


où $(t, \lambda, u, \mu) \mapsto \mu(t, \lambda, u)$ est une application analytique définie dans un voisinage de $\left(t_{0}, \lambda_{0}, u_{0}\right) ; \mu(t, \lambda, u)$ sera une valeur propre simple de $H_{+}+\Gamma_{+}(t, \lambda, u)$. De plus, il existe un vecteur propre unitaire $\varphi(t, \lambda, u)$ associé à $\mu(t, \lambda, u)$ qui est analytique en $(t, \lambda, u)$.

Comme $H_{+}+\Gamma_{+}(t, \lambda, u)$ est auto-adjoint quand $(t, \lambda, u)$ sont réels, on sait que $k(t, \lambda, u, \mu)$ est réelle pour $(t, \lambda, u, \mu)$ réels.

Remarque. - Il n'est pas difficile de voir que, de façon générique, on peut supposer que l'on se trouve dans le cas d'une valeur propre simple.

\subsection{Prolongement de la résolvante de l'opérateur perturbé.}

On rajoute un point $\overline{0}$ à $\mathcal{R}_{\epsilon}$ et on prolonge $\widetilde{S}$ par continuité à ce point en posant $\widetilde{S}(\overline{0})=0$. La façon de rajouter ce point dépend de la singularité de $S$ en 0 .

Considérons

$$
\widetilde{\mathcal{R}}_{\epsilon}:=\widetilde{S}^{-1}\left(D_{\mathbb{C}}(0, \epsilon)\right) .
$$

C'est un ouvert de $\mathcal{R}_{\epsilon}$. On peut alors naturellement prolonger analytiquement $R_{-}$comme opérateur borné de $\mathcal{A}_{0}$ dans $\mathcal{A}_{1}^{*}$ et $L_{+}$comme perturbation $H_{+}$-compacte de $H_{+}$à $\widetilde{\mathcal{R}}_{\epsilon}$ de la façon suivante : pour $z \in \widetilde{\mathcal{R}}_{\epsilon}$,

$$
R_{-}(t, z)=R_{-}(t, \rho(z), \widetilde{S}(z)) \quad \text { et } \quad L_{+}(t, z)=H_{+}+\Gamma_{+}(t, \rho(z), \widetilde{S}(z))
$$

Le fait que, pour $t$ assez petit, $R_{-}(t, \lambda)$ se prolonge holomorphiquement et non méromorphiquement c'est-à-dire que $R_{-}(t, \lambda)$ n'ait pas de pôles au voisinage de 0 , est dû à ce que $1-F(0) \Pi_{-} A(0) \Pi_{-}$est inversible.

De la Proposition 1.1, on déduit immédiatement le :

ThÉorème 1.2. - Supposons que (H.1)-(H.3) soient vérifiées. Il existe un $\epsilon>0$ tel que pour $t \in B(0, \epsilon)$, on peut construire un prolongement holomorphe de $L_{+}(t, z)$ à $\widetilde{\mathcal{R}}_{\epsilon}$ (en tant que perturbation $H_{+}$-compacte de $\left.H_{+}\right)$et $R(t, z)$, un prolongement méromorphe de $(\lambda-H(t))^{-1} \dot{a} \widetilde{\mathcal{R}}_{\epsilon}$ (en tant qu'opérateur borné de $\mathcal{A}_{0}$ dans $\mathcal{A}_{1}^{*}$ ).

De plus, $z$ est un pôle de $R(t, z)$ si et seulement si $\rho(z)$ est valeur propre de $L_{+}(t, z)$.

DÉfinition 1.3. - Les pôles de $R(t, z)$ sont appelés les résonances de $H(t)$.

On remarquera qu'ici on ne distingue pas les résonances des valeurs propres. On dira qu'une résonance est simple si le résidu de $R(t, z)$ à ce pôle est de rang 1 , c'est-à-dire si $\rho(z)$ est une valeur propre simple de $L_{+}(t, z)$. 
DÉFINITION 1.4. - On dira qu'une fonction $\phi$ est une fonction résonante pour $H(t)$ associée à la résonance $z$ si $\phi$ est dans le noyau de $L_{+}(t, z)-\rho(z)$. Soit $\mathbb{E} \subset \widetilde{\mathcal{R}}_{\epsilon}$. On appellera espace résonant associé à $\mathbb{E}$ et à $H(t)$, la somme directe des espaces propres géneralisés associés à $L_{+}(t, z)$ et $\rho(z)$ pour $\lambda \in \mathbb{E}$.

Remarque. - Notons que cette étude n'a d'intérêt que si

$$
\left[A(t), \Pi_{+}\right] \neq 0 \neq\left[A(t), \Pi_{-}\right]
$$

En effet, dans le cas contraire, le système d'équations (1.1) est découplé. Comme $R_{-}(t, \lambda)$ se prolonge holomorphiquement, la seconde équation ne crée pas de pôles et la première crée au plus des valeurs propres qui peuvent être plongées dans le spectre essentiel de $H$. Les éventuelles résonances proviennent du couplage entre $A(t)$ et $H$.

Preuve du Théorème 1.2. - D'après (1.2) et la Proposition 1.1, pour $\left.\left.\lambda \in D_{\mathbb{C}}\left(0, \epsilon_{0}\right) \backslash\right]-\epsilon_{0}, 0\right]$, pour calculer $(\lambda-H(t))^{-1} \psi$ pour $\psi \in \mathcal{A}_{0}$, il suffit de pouvoir inverser $L_{+}(t, \lambda)-\lambda$ sur $\mathcal{H}$. On remarque que :

$$
L_{+}(t, \lambda)-\lambda=\left(1+\Gamma_{+}(t, \lambda, S(\lambda))\left(H_{+}-\lambda\right)^{-1}\right)\left(H_{+}-\lambda\right) .
$$

Or, pour $\lambda$ voisin de 0 , on a $\lambda \notin \sigma\left(H_{+}\right)$(par la remarque suivant l'hypothèse (H.1)) et :

$$
\left\|\left(H_{+}-\lambda\right)^{-1}\right\|_{\mathcal{B}(\mathcal{H})} \leq \frac{1}{\left|\epsilon_{0}-\lambda\right|} .
$$

Comme $\Gamma_{+}(t, \lambda, S(\lambda))$ est $H_{+}$-compact par la Proposition $1.1, L_{+}(t, \lambda)-\lambda$ est inversible si et seulement si $\lambda$ n'est pas valeur propre de $L_{+}(t, \lambda)$. En utilisant alors les prolongements analytiques construits pour $R_{-}$et $L_{+}$, on obtient le Théorème 1.2.

\section{2. Éclatement des résonances}

\subsection{Différents types de ramification.}

Nous étudions l'éclatement d'une résonance $z_{0}$ de $H(0)$ en résonances de $H(t)$ quand $t$ varie au voisinage de 0 .

Le cas où $z_{0}$ n'est pas au seuil, c'est-à-dire si $\rho\left(z_{0}\right) \neq 0$, est traité en 2.2 . Le cas très intéressant où $z_{0}=\overline{0}$, le point rajouté à $\mathcal{R}_{\epsilon}$ est plus délicat. Pour l'étudier il faut préciser la manière dont on peut ajouter un point à $\mathcal{R}_{\epsilon}$. On le fait pour deux types de singularités de $S$ :

a) type rationnel $S(z)=z^{p / q}$ avec $p / q$ rationnel positif non entier,

b) type logarithmique $S(z)=z^{p} \cdot \log (z)$ avec $p \in \mathbb{N}^{*}$.

(On considère ici les branches principales de la racine $q$-ième et du logarithme).

TоMе $124-1996-\mathrm{N}^{\circ} 3$ 
Remarque. - Rappelons que dans les applications à Schrödinger dans $\mathbb{R}^{n}$, on a :

- $S(\lambda)=\lambda^{(n-2) / 2}$ pour $n$ impair (type rationnel), et

- $S(\lambda)=\lambda^{(n-2) / 2} \log \lambda$ pour $n$ pair (type logarithmique).

\subsection{Cas où la résonance n'est pas au seuil.}

Supposons que (H.1)-(H.3) soient vérifiées. Supposons que $z_{0} \in \widetilde{\mathcal{R}}_{\epsilon}$ est une résonance de $H\left(t_{0}\right)$. Par définition, $\rho\left(z_{0}\right)$ est alors une valeur propre de

$$
L_{+}\left(t_{0}, z_{0}\right)=H_{+}+\Gamma_{+}\left(t_{0}, \rho\left(z_{0}\right), \widetilde{S}\left(z_{0}\right)\right) .
$$

Donc, il existe $h(t, z)$, une fonction analytique $h(t, z)$ sur un voisinage $U \times V$ de $\left(t_{0}, z_{0}\right)$, telle que :

1) $h\left(t_{0}, z_{0}\right)=0$

2) $\operatorname{pour}(t, z) \in U \times V,(z$ est une résonance de $H(t)) \Leftrightarrow(h(t, z)=0)$.

Remarquons que si $\partial_{z} h(t, z)_{\mid z_{0}} \neq 0$, alors on peut trouver une application analytique $t \mapsto z(t)$ d'un voisinage de $t_{0}$ dans un voisinage de $z_{0}$ tel que $z(t)$ est l'unique résonance de $H(t)$ dans le voisinage de $z_{0}$ considéré. Dans le cas où $z_{0}$ est une résonance simple de $H\left(t_{0}\right)$, pour $t$ voisin de $t_{0}$, il n'y a qu'une résonance de $H(t)$ voisine de $z_{0}$ et celle-ci est simple.

REMARQUe. - Ce cas recouvre d'une certaine manière celui traité par A. Orth [Or].

2.3. Résonance en un seuil de type rationnel : $S(z)=z^{p / q}$.

Il est classique qu'on obtient une surface de Riemann régulière en rajoutant un point à $\mathcal{R}_{\epsilon}$. Plus précisément, on définit :

$$
\mathcal{R}(q, \epsilon)=\left\{\zeta=\left(z, z^{q}\right) ; z \in D(0, \epsilon)\right\} \subset \mathbb{C}^{2},
$$

le graphe de la puissance $q$-ième restreinte à $D(0, \epsilon)$. Alors :

$$
\mathcal{R}_{\epsilon}=\mathcal{R}(q, \epsilon) \backslash(0,0) \text { et } \quad \overline{0}=(0,0)
$$

L'application $\rho$ est définie $\operatorname{par} \rho(z)=z^{q}$ et $\widetilde{S}(z)=z^{p}$ (ici on utilise l'identification $\left.\rho_{1}: \zeta \in \mathcal{R}(q, \epsilon) \leftrightarrow z \in D(0, \epsilon)\right)$.

On prolonge alors la résolvante $R(t, \zeta)$ à $\mathcal{R}_{\epsilon}: z \in \mathcal{R}_{\epsilon}$ sera une résonance de $H(t)$ si et seulement si $z^{q}$ est une valeur propre de $H_{+}+\Gamma_{+}\left(t, z^{q}, z^{p}\right)$.

Supposons que $\overline{0}$ est une résonance de $H(0)$, c'est-à-dire que $0=\rho(\overline{0})$ est une valeur propre de $H_{+}+\Gamma_{+}(0,0,0)$. Ainsi, il existe une équation aux valeurs propres $k(t, \lambda, u, \mu)=0$ avec $k$ analytique telle que : 
(i) $k(0,0,0,0)=0$,

(ii) pour $(t, \lambda, u, \mu) \in D(0, \epsilon)^{4}, \mu$ est une valeur propre de $H_{+}+$ $\Gamma_{+}(t, \lambda, u)$ si et seulement si $k(t, \lambda, u, \mu)=0$.

On obtient donc l'équation aux résonances suivante pour $H(t)$,

$$
h(t, z):=k\left(t, z^{q}, z^{p}, z^{q}\right)=0,
$$

c'est-à-dire, en tenant compte de l'analyticité de $k$,

$$
h(t, z)=a_{1,0,0} t+a_{0,1,0} z^{q}+a_{0,0,1} z^{p}+\sum_{\substack{j+k+\ell \geq 2 \\ j, k, \ell \geq 0}} a_{j, k, \ell} t^{j} z^{q k+p \ell}=0 .
$$

Rappelons que les coefficients $\left(a_{j, k, \ell}\right)_{j, k, \ell}$ sont réels.

Hypothèse (H.4). - $a_{1,0,0} \neq 0, a_{0,1,0} \neq 0$ et $a_{0,0,1} \neq 0$.

Remarque. - Les trois hypothèses de (H.4) ne sont pas toutes nécessaires en même temps. En effet comme le montre la preuve du Théorème 2.1, si $p>q$, il suffit de supposer $a_{1,0,0} \neq 0$ et $a_{0,1,0} \neq 0$. Et si $p<q$, il suffit de supposer $a_{1,0,0} \neq 0$ et $a_{0,0,1} \neq 0$.

Alors, on extrait les racines $z(t)$ de l'équation (2.1) et on obtient le :

ThÉorème 2.1. - Soit $m=\min (p, q)$. Supposons que $\overline{0}$ soit une résonance de $H(0)$ et que les hypothèses (H.1)-(H.4) soient vérifiées. Alors, il existe $m$ résonances de $H(t)$ près de $\overline{0}$ pour $t \neq 0$. Plus précisément, il existe $\epsilon_{1}>0, \epsilon_{2}>0$ et une fonction analytique $f$ de $D\left(0, \epsilon_{1}\right)$ dans $D\left(0, \epsilon_{2}\right)$ (dont les coefficients de Taylor sont réels et telle que $f(0)=0$ ) tels que si on définit

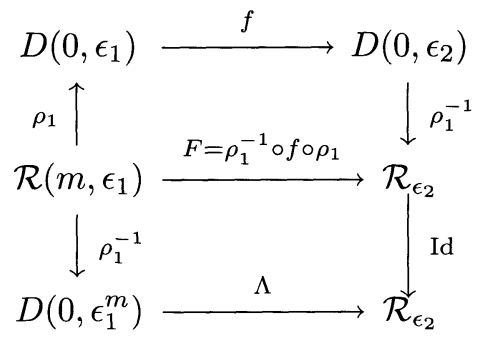

alors, pour $t \in D\left(0, \epsilon_{1}^{m}\right)$,

$$
\Lambda(t)=F\left(\rho^{-1}(t)\right)=\left\{\lambda_{0}(t), \ldots, \lambda_{m-1}(t)\right\}
$$

est l'ensemble des résonances de $H(t)$ contenues dans $\mathcal{R}_{\epsilon_{2}}$.

Preuve du Théorème 2.1. - On peut supposer sans restrictions que $m=p\left(\right.$ i.e. $p<q$ ). On va résoudre (2.1). Pour cela, on pose $t=u^{p}$. On a le :

TOME $124-1996-\mathrm{N}^{\circ} 3$ 
LEMME 2.2. - Supposons qu'il existe une application continue $u \mapsto z(u)$ au voisinage de 0 et telle que $z(0)=0$ qui vérifie $h\left(u^{p}, z(u)\right)=0$ pour $u$ voisin de 0 . Alors, il existe $k \in\{0,1, \ldots, p-1\}$ tel que

$$
z(u)=u \cdot \mathrm{e}^{2 i \pi \frac{k}{p}} \delta_{0}(1+\mathrm{o}(1))
$$

quand $u$ tend vers 0 et où $\delta_{0} \in \mathbb{C}$ vérifie $\delta_{0}^{p}=-a_{1,0,0} / a_{0,0,1}$.

Preuve. - En substituant $z(u)$ dans (2.1), on obtient :

$$
a_{1,0,0} u^{p}(1+\alpha(u))=-a_{0,0,1} z(u)^{p}(1+\beta(u)),
$$

où $\alpha$ et $\beta$ sont continues au voisinage de 0 et $\alpha(0)=\beta(0)=0$. Donc

$$
\left(\frac{z(u)}{\delta_{0} u}\right)^{p}=(1+\gamma(u))
$$

où $\gamma(u)$ est continue au voisinage de $0, \gamma(0)=0$ et $\delta_{0}^{p}=-a_{1,0,0} / a_{0,0,1}$. Ainsi, pour $u$ voisin de 0 non nul,

$$
z(u)=\delta_{0} \mathrm{e}^{2 i \pi \frac{k(u)}{p}} u \sqrt[p]{1+\gamma(u)} \text { où } k(u) \in\{0, \ldots, p-1\} .
$$

Donc, comme $u \mapsto z(u)$ est supposée continue au voisinage de 0 , l'application $u \mapsto k(u)$ est constante sur un voisinage de 0 . Ceci achève la preuve du Lemme 2.2 .

On va maintenant résoudre $(2.1)$ en prenant $t=u^{p}$ et en cherchant $z$ sous la forme suivante

$$
z=\delta_{k} \cdot u \cdot(1+f(u)) \text { où } \quad \delta_{k}=\mathrm{e}^{2 i \pi \frac{k}{p}} \delta_{0} .
$$

En effet par le Lemme 2.2, on sait que toute les solutions de (2.1) continue au voisinage de 0 et valant 0 en 0 sont de cette forme. En substituant cette expression dans (2.1), on obtient l'équation

$$
\begin{aligned}
a_{1,0,0}\left(1-(1+f(u))^{p}\right) u^{p}+a_{0,1,0} \delta_{k}^{q}(1+f(u))^{q} u^{q} & \\
& +\sum_{\substack{j+k+\ell \geq 2 \\
j, k, \ell \geq 0}} a_{j, k, \ell} u^{p(j+\ell)+q k}\left(\delta_{k}(1+f(u))\right)^{q k+p \ell}=0
\end{aligned}
$$

soit encore pour $u \neq 0$,

$$
g_{k}(u, f(u))=0
$$


avec

$$
\begin{aligned}
g_{k}(u, f)=a_{1,0,0}(1 & \left.-(1+f)^{p}\right)+a_{0,1,0} \delta_{k}^{q}(1+f)^{q} u^{q-p} \\
& +\sum_{\substack{j+k+\ell \geq 2 \\
j, k, \ell \geq 0}} a_{j, k, \ell} u^{p(j+\ell-1)+q k}\left(\delta_{k}(1+f)\right)^{q k+p \ell} .
\end{aligned}
$$

On a $g_{k}(0,0)=0$ et $\partial_{f} g_{k}(0,0)=-p a_{1,0,0} \neq 0$. Donc, par le théorème des fonctions implicites holomorphes, il existe une application analytique $u \mapsto f_{k}(u)$, définie sur $U$, un voisinage de 0 , et telle que, pour $u \in U$, $g_{k}\left(u, f_{k}(u)\right)=0$. On peut remarquer que

$$
\begin{gathered}
0=g_{0}\left(\mathrm{e}^{2 i \pi \frac{k}{p}} u, f_{0}\left(\mathrm{e}^{2 i \pi \frac{k}{p}} u\right)\right)=g_{k}\left(u, f_{0}\left(\mathrm{e}^{2 i \pi \frac{k}{p}} u\right)\right)=g_{k}\left(u, f_{k}(u)\right) \\
f_{k}(0)=0=f_{0}(0)
\end{gathered}
$$

Ainsi, on conclut par l'unicité dans le théorème des fonctions implicites, que $f_{k}(u)=f_{0}\left(\mathrm{e}^{2 i \pi \frac{k}{p}} u\right)$

Finalement, on a obtenu que les solutions $z(t)$ de (2.1) sont de la forme

$$
z(t)=\delta_{0} u\left(1+f_{0}(u)\right)
$$

avec $t=u^{p}$ pour $t$ dans un voisinage de 0 dans $\mathbb{C}$.

Projetés des résonances.

Les projetés sur la base de $\mathcal{R}\left(q, \epsilon_{2}\right)$ des résonances obtenues dans le Théorème 2.1 sont, pour $0 \leq k \leq m-1$,

$$
\lambda_{k}(t)=f_{1} \cdot \mathrm{e}^{2 i \pi \frac{k q}{m}} t^{\frac{q}{m}}\left(1+\tilde{f}\left(\mathrm{e}^{2 i \pi \frac{k}{m}} t^{\frac{1}{m}}\right)\right)^{q},
$$

si $f(z)=f_{1} \cdot z \cdot(1+\tilde{f}(z))$ où $\tilde{f}$ est analytique au voisinage de 0 et $\tilde{f}(0)=0$. Ainsi, si $q<p$ (i.e. $m=q)$, le comportement des résonances est linéaire en $t$ alors qu'il est de type puissance fractionnaire si $p<q$.

Discussion.

On a supposé $q>1$.

- Si $m=p=1$, on obtient pour $t$ petit une unique résonance simple au voisinage du seuil.

- Par contre si $m>1$, il apparaît plusieurs résonances; plus exactement, si $1<q<p$, il apparaît exactement une résonance sur chaque feuillet de $\mathcal{R}_{\epsilon_{2}}$, et si $1<p<q$, alors il n'apparaît de résonances que sur certains feuillets de $\mathcal{R}_{\epsilon_{2}}$. Remarquons que le phénomène d'éclatement ne provient pas de la multiplicité de la résonance en $\overline{0}$, c'est-à-dire que même si cette résonance est simple, il y a un éclatement.

TOME $124-1996-\mathrm{N}^{\circ} 3$ 


\subsection{Résonance en un seuil de type logarithmique :} $\boldsymbol{S}(\boldsymbol{z})=\boldsymbol{z}^{\boldsymbol{p}} \cdot \log (\boldsymbol{z})$ avec $\boldsymbol{p} \in \mathbb{N}^{*}$.

Dans ce cas-ci, le problème vient de ce que $z^{p} \cdot \log (z)$ admet une singularité essentielle en 0 en plus de la ramification. Pour uniformiser $S$, on utilise le revêtement universel que l'on utilise pour uniformiser le logarithme. Soit

$$
\widetilde{\mathcal{R}}_{\epsilon}=\left\{\zeta=\left(z, \mathrm{e}^{z}\right) ; z \in \mathbb{C} \text { et } \operatorname{Re}(z)<\log \epsilon\right\} .
$$

On uniformise $S(z)=z^{p} \cdot \log z$ de la façon suivante :

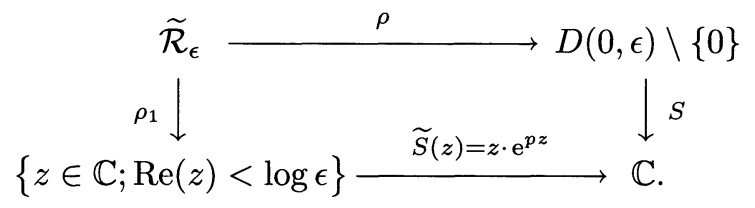

Ici $\rho_{1}$ est la projection sur la première coordonnée et $\rho$ celle sur la seconde. On va maintenant restreindre $\widetilde{\mathcal{R}}_{\epsilon}$ à un ouvert de façon à ce que l'uniformisation $\widetilde{S}$ de $S$ sur cet ouvert soit à valeurs dans $D(0, \epsilon)$ et de plus puisse être prolongée par continuité au point $(-\infty, 0)$ sur cet ouvert. Soit $\nu>0$. On définit :

$$
\mathcal{R}_{\epsilon, \nu}=\left\{\zeta=\left(z, \mathrm{e}^{z}\right) \in \widetilde{\mathcal{R}}_{\epsilon} ;(p+\nu) \cdot \operatorname{Re}(z)+|z|<\log \epsilon\right\} .
$$

On vérifie aisément que :

(i) $\mathcal{R}_{\epsilon, \nu}$ est un ouvert de $\widetilde{\mathcal{R}}_{\epsilon}$;

(ii) $\left|\widetilde{S} \circ \rho_{1}(\zeta)\right|<\epsilon$ pour $\zeta \in \mathcal{R}_{\epsilon, \nu}$;

(iii) pour $\zeta \in \mathcal{R}_{\epsilon, \nu}, \widetilde{S} \circ \rho_{1}(\zeta)$ tend vers 0 si $\operatorname{Re}\left(\rho_{1}(\zeta)\right)$ tend vers $-\infty$.

On peut alors compactifier $\mathcal{R}_{\epsilon, \nu}$ en lui ajoutant le point $\overline{0}=(-\infty, 0)$ et prolonger $\widetilde{S} \circ \rho_{1}$ par continuité à cette compactification par $\widetilde{S} \circ \rho_{1}(\overline{0})=0$. Dans ce cas-ci, $\overline{0}$ est le point de ramification. On définit :

$C_{\epsilon, \nu}=\rho_{1}\left(\mathcal{R}_{\epsilon, \nu}\right)=\{z \in \mathbb{C} ; \operatorname{Re}(z)<\log \epsilon$ et $(p+\nu) \cdot \operatorname{Re}(z)+|z|<\log \epsilon\}$.

Supposons que $\overline{0}$ est une résonance de $H(0)$; donc $0=\rho(\overline{0})$ est une valeur propre de $H_{+}+\Gamma_{+}(0,0,0)$. Ainsi, il existe une équation aux valeurs propres $k(t, \lambda, u, \mu)=0$ avec $k$ analytique telle que

(i) $k(0,0,0,0)=0$;

(ii) pour $(t, \lambda, u, \mu) \in D(0, \epsilon)^{4}, \mu$ est une valeur propre de

$$
H_{+}+\Gamma_{+}(t, \lambda, u)
$$

si et seulement si $k(t, \lambda, u, \mu)=0$. 
Donc, pour $t \in D(0, \epsilon), \zeta \in \mathcal{R}_{\epsilon, \nu}$ est une résonance de $H(t)$ si et seulement si

$$
k(t, \rho(\zeta), S \circ \rho(\zeta), \rho(\zeta))=0
$$

Donc en utilisant le développement en série entière de $k$ au voisinage de $(0,0,0,0)$ et les définitions de $S$ et $\rho,(2.3)$ devient :

$$
\begin{gathered}
h(t, z)=a_{1,0,0} t+a_{0,1,0} z e^{p z} \mathrm{e}^{z} \\
+a_{0,0,1}+\sum_{\substack{j+k+\ell \geq 2 \\
j, k, \ell \geq 0}} a_{j, k, \ell} t^{j} z^{k} \mathrm{e}^{(p k+\ell) z}=0 .
\end{gathered}
$$

Rappelons que les coefficients $\left(a_{j, k, \ell}\right)_{j, k, \ell}$ sont réels.

HYpothèSE (H.4 bis). - $a_{1,0,0} \neq 0, a_{0,1,0} \neq 0$ et $a_{0,0,1} \neq 0$.

REMARQUE. - La remarque faisant suite à l'hypothèse (H.4) s'applique ici, à la nuance suivante près : il suffit de supposer

- $a_{1,0,0} \neq 0$ et $a_{0,1,0} \neq 0$ si $p=1$ et

- $a_{1,0,0} \neq 0$ et $a_{0,0,1} \neq 0$ si $p>1$.

THÉORÈmE 2.3. - Supposons que $\overline{0}$ est une résonance de $H(0)$ et supposons les hypothèses (H.1)-(H.4 bis) vérifiées. Alors, asymptotiquement, il existe une infinité de résonances au voisinage de $\overline{0}$. Plus précisément, pour $\nu>0$, il existe $\epsilon_{1}>0, \epsilon_{2}>0$ et $f$, une fonction analytique de $C_{\epsilon_{1}, \nu}$ dans $C_{\epsilon_{2}, \nu}$ tels que si on définit

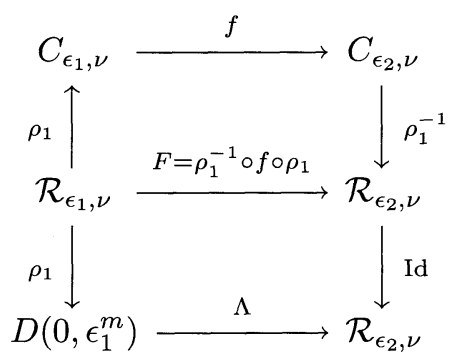

est l'ensemble des résonances de $H(t)$ contenues dans $\mathcal{R}_{\epsilon_{2}, \nu}$.

De plus, on a les asymptotiques suivantes sur $f$ :

(i) si $p>1$, pour $\delta$ défini par $\mathrm{e}^{\delta}=\mathrm{a}_{1,0,0} / \mathrm{a}_{0,0,1}$ et $0 \leq \operatorname{Im}(\delta) \leq \pi$, on $a$ :

$$
f(z)=z+\delta+h_{p}\left(z^{-1}, z \mathrm{e}^{(p-1) z} \mathrm{e}^{z}\right)
$$

TOME $124-1996-\mathrm{N}^{\circ} 3$ 
(ii) si $p=1$, pour $\delta$ défini par $\mathrm{e}^{\delta}=a_{1,0,0} / a_{0,1,0}$ et $0 \leq \operatorname{Im}(\delta) \leq \pi$, on $a$ :

$$
f(z)=z-\log (-z)+\delta+h_{1}\left(z^{-1}, z^{-1} \log (-z), \mathrm{e}^{z}\right)
$$

où $\log$ est la branche principale du logarithme et les fonctions $h_{p}$ sont analytiques au voisinage de 0 dans toutes leurs variables et $h_{p}(0,0,0)=0$.

Remarque. - La forme de $C_{\nu}(\epsilon)$ implique que $n(t)$ tend vers $+\infty$ quand $t$ tend vers 0 .

Preuve du théorème 2.3. - On va procéder comme dans la preuve du Théorème 2.1. On prouvera d'abord le :

LEMME 2.4. - Supposons qu'il existe une application continue $u \mapsto z(u)$ au voisinage de $-\infty$ dans $C_{\epsilon, \nu}$ (où $\nu>0$ et $\epsilon>0$ sont fixés) et telle que:

(i) $\operatorname{Re}(u) \rightarrow-\infty$ quand $\mathrm{e}^{z(u)} \rightarrow 0$

(ii) $h\left(\mathrm{e}^{u}, z(u)\right)=0$ pour $\operatorname{Re}(u)$ assez négatif.

Alors, il existe $k \in \mathbb{Z}$ tel que :

(i) si $p>1, z(u)=u+\delta+2 i k \pi+\mathrm{o}(1)$ pour $\operatorname{Re}(u)$ assez négatif,

(ii) si $p=1, z(u)=u-\log (-u)+\delta+2 i k \pi+\mathrm{o}(1)$ pour $\operatorname{Re}(u)$ assez négatif, où $\log$ est la branche principale du logarithme et $\delta$ est défini dans le Théorème 2.3.

Preuve.

- Si $p>1$, comme $z \mathrm{e}^{p z}$ est négligeable devant $\mathrm{e}^{z}$ au voisinage de $-\infty$, la preuve du Lemme 2.4 est exactement celle du Lemme 2.2.

- Si $p=1$, en substituant $z(u)$ et $t=\mathrm{e}^{u}$ dans (2.4), on obtient :

$$
a_{1,0,0} \mathrm{e}^{u}(1+\alpha(u))=a_{0,0,1}(-z(u)) \mathrm{e}^{z(u)}(1+\beta(u)),
$$

où $\alpha(u) \rightarrow 0$ et $\beta(u) \rightarrow 0$ quand $\operatorname{Re}(u) \rightarrow-\infty$. En prenant le logarithme de la formule précédente, on obtient pour $\operatorname{Re}(u)$ suffisamment négatif

$$
u+\delta=z(u)+\log (-z(u))+2 i k(u) \pi+\mathrm{o}(1)
$$

où $o(1) \rightarrow 0$ quand $\operatorname{Re}(u) \rightarrow-\infty$ et $k(u) \in \mathbb{Z}$ pour tout $u$. Donc, pour $\left(u, u^{\prime}\right)$ assez négatifs,

$$
\left|k(u)-k\left(u^{\prime}\right)\right|<\frac{1}{2}+C\left(\left|u-u^{\prime}\right|+\left|z(u)-z\left(u^{\prime}\right)\right|\right) .
$$

Comme $z$ est supposée continue et que $u \mapsto k(u)$ ne prend que des valeurs entières, $u \mapsto k(u)$ est localement constante au voisinage de $-\infty$, donc constante comme nous considérons des voisinages connexes. Alors, par (2.5), on obtient $u \sim z(u)$ quand $\operatorname{Re}(u) \rightarrow-\infty$. On achève la preuve du Lemme 2.4 en réutilisant (2.5). 
On ne traitera explicitement que le cas $p=1$, le cas $p>1$ étant plus simple à traiter. On va résoudre (2.4) en prenant $t=\mathrm{e}^{u}$ et en cherchant $z$ sous la forme $z=u+\delta-\log (-u)+g(u)$ où $\delta$ est défini dans le Théorème 2.3 En substituant ces expressions dans (2.4), on obtient :

$$
\begin{aligned}
a_{1,0,0} \mathrm{e}^{u} & -a_{1,0,0}\left(1-u^{-1} \log (-u)+u^{-1}(\delta+g)\right) \mathrm{e}^{u} \mathrm{e}^{g} \\
& +a_{0,0,1} \mathrm{e}^{u} u^{-1} \mathrm{e}^{\delta+g} \\
& +\sum_{\substack{j+k+\ell \geq 2 \\
j, k, \ell \geq 0}} a_{j, k, \ell} \mathrm{e}^{(k+\ell) \delta} \mathrm{e}^{(j+k+\ell) u} u^{-\ell} \\
& \left.=H\left(1-u^{-1} \log (-u)+(\delta+g) u^{-1}\right)^{k} \mathrm{e}^{(k+\ell) g}, u^{-1} \log (-u), \mathrm{e}^{u}\right)=0,
\end{aligned}
$$

où $(g, a, b, c) \mapsto H(g, a, b, c)$ est une application analytique au voisinage de $(0,0,0,0)$ qui vérifie $H(0,0,0,0)=0$ et $\partial_{g} H(0,0,0,0)=-a_{1,0,0}$. Par le théorème des fonctions implicites, on sait qu'il existe une fonction analytique $(a, b, c) \mapsto g(a, b, c)$ définie sur un voisinage de $(0,0,0)$ telle que $g(0,0,0)=0$ et $H(g, a, b, c)=0$ si et seulement si $g=g(a, b, c)$.

Pour résoudre (2.6), pour $u \in C_{\nu, \epsilon}$ et $\operatorname{Re}(u)$ assez négatif, on pose :

$$
z_{0}(u)=u+\delta-\log (-u)+g\left(u^{-1}, u^{-1} \log (-u), \mathrm{e}^{u}\right) .
$$

Remarquons que pour $k \in \mathbb{Z}, z_{k}(u)=z_{0}(u+2 i k \pi)$ est aussi solution de (2.4) (si $u$ vérifie $u+2 i k \pi \in C_{\epsilon, \nu}$ ).

Soit $u \mapsto w(u)$ une application continue au voisinage $-\infty$ dans $C_{\epsilon, \nu}$ (où $\nu>0$ et $\epsilon>0$ fixés) et telle que :

(i) $w(u) \rightarrow 0$ quand $\operatorname{Re}(u) \rightarrow-\infty$;

(ii) $h\left(\mathrm{e}^{u}, w(u)\right)=0$ pour $\operatorname{Re}(u)$ assez négatif.

Alors par le Lemme 2.4, il existe $k \in \mathbb{Z}$ tel que $w(u)-z_{k}(u)=\mathrm{o}(1)$ quand $\operatorname{Re}(u)$ tend vers $-\infty$. Donc, il existe $\theta(u) \in] 0,1[$ tel que

$$
\begin{aligned}
0 & =h\left(\mathrm{e}^{u}, w(u)\right)-h\left(\mathrm{e}^{u}, z_{k}(u)\right) \\
& =\left(w(u)-z_{k}(u)\right) \cdot \partial_{2} h\left(\mathrm{e}^{u}, z_{k}(u)+\theta(u)\left(w(u)-z_{k}(u)\right)\right) .
\end{aligned}
$$

Quand $\operatorname{Re}(u)$ tend vers $-\infty$, on voit aisément que

$$
\partial_{2} h\left(\mathrm{e}^{u}, z_{k}(u)+\theta(u)\left(w(u)-z_{k}(u)\right)\right)=z_{k}(u) \mathrm{e}^{-z_{k}(u)} \cdot(1+\mathrm{o}(1)) .
$$

On en conclut que $w(u)=z_{k}(u)$ pour $\operatorname{Re}(u)$ assez négatif. On a ainsi obtenu toutes les solutions de l'équation (2.4) en posant $z(t)=z_{0}(u)$ pour $t=\mathrm{e}^{u}$. Ceci achève la preuve du Théorème 2.3. 


\section{Conclusion}

On a pu définir un prolongement méromorphe de la résolvante, sous des hypothèses assez générales. Ceci s'applique en particulier au cas de l'opérateur de Schrödinger périodique auquel on ajoute une perturbation à support compact. Pour l'opérateur de Schrödinger périodique, le prolongement de la résolvante est construit dans [Gé2]. Au voisinage de l'extrémité supérieure d'une bande spectrale simple (il suffit que le bord de la bande que nous considérons soit simple, et que le seuil soit un extremum non dégénéré de la fonction de bande), notre hypothèse (H.1) est satisfaite en dimension $n \geq 3$. Pour fixer les idées, supposons que cette extrémité est 0 . Si on considère une perturbation de la forme $A(t)=t W$ où $W$ est un potentiel non-négatif qui décroît suffisamment vite à l'infini (typiquement, une décroissance exponentielle) et $t_{0}<0$, notre hypothèse (H.3) est satisfaite pour $t$ voisin de $t_{0}$.

Supposons que lorsque $t$ décroît vers $t_{0}$, il existe une valeur propre $\lambda(t)$ de $H(t)$ qui tend vers 0 . Alors en utilisant l'équation aux valeurs propres pour l'opérateur de Livsic dans ce cas, on a, pour $t>t_{0}$ assez proche de $t_{0}$,

$$
k(t, \lambda(t), S(\lambda(t)), \lambda(t))=0 .
$$

Comme $S$ et $k$ sont continues, on a $k\left(t_{0}, 0,0,0\right)=0$. On a donc une résonance au seuil pour $t=t_{0}$.

Vérifions que les hypothèses (H.4) et (H.4 bis) sont génériquement satisfaites dans le cas présent. Ici, génériquement s'entend pour $A$ dans la classe des perturbations vérifiant (H.3); entre autres $A$ peut ne pas être un potentiel. Génériquement, la valeur propre 0 de $H_{+}+\Gamma_{+}\left(t_{0}, 0,0\right)$ peut être supposée simple; on note $\mu(t, \lambda, u)$ la branche de valeur propre correspondante.

Pour prouver que $a_{1,0,0} \neq 0$, génériquement, il suffit de perturber $A$ par un projecteur choisi convenablement. En effet, comme $\mu(t, \lambda, u)$ est simple de $H_{+}+\Gamma_{+}(t, \lambda, u)$, on peut lui associer $\varphi(t, \lambda, u)$, un vecteur propre unitaire analytique en $(t, \lambda, u)$. De plus, $\Gamma_{+}$est $H_{+}$-compact de $\mathcal{H}_{0}$ à valeurs dans $\mathcal{A}_{0}$. Donc, comme 0 n'est pas dans le spectre de $H_{+}$, on a $\varphi(t, \lambda, u) \in \Pi_{+}\left(\mathcal{A}_{1}\right) \subset \mathcal{A}_{1}$.

Supposons $a_{1,0,0}=\partial_{t} \mu\left(t_{0}, 0,0\right)=0$. Soit $P_{+}$, le projecteur orthogonal sur $\varphi\left(t_{0}, 0,0\right)$ et, pour $\epsilon$ petit réel,

$$
A^{\epsilon}(t)=A(t)+\epsilon\left(t-t_{0}\right) P_{+} .
$$

Alors $A^{\epsilon}(t)$ vérifie (H.3) et on peut étudier l'opérateur $H+A^{\epsilon}(t)$. Comme dans le cas $\epsilon=0$, on lui associe :

$$
\Gamma_{+}^{\epsilon}(t, \lambda, u)=\Gamma_{+}(t, \lambda, u)+\epsilon\left(t-t_{0}\right) P_{+} .
$$


Donc, pour $\epsilon$ assez petit, $H_{+}+\Gamma_{+}^{\epsilon}(t, \lambda, u)$ admet une unique valeur propre simple voisine de 0 que nous noterons $\mu^{\epsilon}(t, \lambda, u)$. L'opérateur $H_{+}+$ $\Gamma_{+}^{\epsilon}(t, \lambda, u)$ est analytique en $(\epsilon, t, \lambda, \mu)$, donc à $\mu^{\epsilon}(t, \lambda, u)$, on peut associer $\varphi^{\epsilon}(t, \lambda, u)$, un vecteur propre unitaire analytique. Comme $\mu^{\epsilon}(t, \lambda, u)_{\mid \epsilon=0}=$ $\mu(t, \lambda, u)$ et $\varphi^{\epsilon}(t, \lambda, u)_{\mid \epsilon=0}=\varphi(t, \lambda, u)$, le calcul de la dérivée de $\mu^{\epsilon}$ nous donne :

$$
\begin{aligned}
a_{1,0,0}^{\epsilon} & =\partial_{t} \mu^{\epsilon}\left(t_{0}, 0,0\right) \\
& =\left\langle\left(\partial_{t} \Gamma_{+}\left(t_{0}, 0,0\right)+\epsilon P_{+}\right) \varphi\left(t_{0}, 0,0\right), \varphi\left(t_{0}, 0,0\right)\right\rangle \\
& =\partial_{t} \mu\left(t_{0}, 0,0\right)+\epsilon=\epsilon \neq 0 .
\end{aligned}
$$

Maintenant, suivant la dimension $n$, nous discutons de la validité des hypothèses $a_{0,1,0} \neq 0$ et $a_{0,0,1} \neq 0$. On fera la discussion dans le cas $n$ impair, donc $S(\lambda)=\lambda^{(n-2) / 2}$. Si $n \geq 5$, alors $n-2>2$; donc il s'agit de voir que $a_{0,1,0} \neq 0$, soit encore $\partial_{\lambda} \mu\left(t_{0}, 0,0\right)-1 \neq 0$. On calcule :

$$
\begin{aligned}
\partial_{\lambda} \Gamma_{+} & \left(t_{0}, 0,0\right) \\
= & A_{+-}\left(t_{0}\right) \partial_{\lambda} R_{-}\left(t_{0}, 0,0\right) A_{-+}\left(t_{0}\right) \\
= & \frac{1}{2}\left\{A _ { + - } ( t _ { 0 } ) \left(\partial_{\lambda} F_{-}(0) U+\right.\right. \\
& F_{-}(0) U A_{--}\left(t_{0}\right) \partial_{\lambda} F_{-}(0) U+U^{*} \partial_{\lambda} F_{-}(0) \\
& \left.\left.\quad+U^{*} \partial_{\lambda} F_{-}(0) A_{--}\left(t_{0}\right) U^{*} F_{-}(0)\right) A_{-+}\left(t_{0}\right)\right\} \\
= & A_{+-}\left(t_{0}\right)\left\{U^{*} \partial_{\lambda} F_{-}(0) U\right\} A_{-+}\left(t_{0}\right)
\end{aligned}
$$

où $U=\left(1-A_{--}\left(t_{0}\right) F_{-}(0)\right)^{-1}$ et en utilisant $1+A_{--}\left(t_{0}\right) U^{*} F_{-}(0)=U$.

Mais, comme $\partial_{\lambda} S(0)=0$, on vérifie aisément que $\partial_{\lambda} F_{-}(0) \leq 0$, ainsi $\partial_{\lambda} \mu\left(t_{0}, 0,0\right) \leq 0$, c'est-à-dire $a_{0,1,0} \neq 0$.

Si $n=3$, comme $n-2<2$, il faut prouver que $a_{0,0,1} \neq 0$, c'est-à-dire $\partial_{u} \mu\left(t_{0}, 0,0\right) \neq 0$. Avec les mêmes notations que ci-dessus, on calcule :

$$
\begin{aligned}
& \partial_{u} \Gamma_{+}\left(t_{0}, 0,0\right)=A_{+-}\left(t_{0}\right) \partial_{u} R_{-}\left(t_{0}, 0,0\right) A_{-+}\left(t_{0}\right) \\
& =\frac{1}{2}\left\{A _ { + - } ( t _ { 0 } ) \left(G_{-}(0) U+F_{-}(0) U A_{--}\left(t_{0}\right) G_{-}(0) U\right.\right. \\
& \left.\left.+U^{*} G_{-}(0)+U^{*} G_{-}(0) A_{--}\left(t_{0}\right) U^{*} F_{-}(0)\right) A_{-+}\left(t_{0}\right)\right\} \\
& =A_{+-}\left(t_{0}\right)\left\{U^{*} G_{-}(0) U\right\} A_{-+}\left(t_{0}\right) \text {. }
\end{aligned}
$$

D'aprè [Gé2, Cor. 3.7 et Cor. 4.2], on sait que si l'extrémité de la bande à laquelle on se place est simple, $G_{-}(0)$ peut s'écrire $G_{-}(0)=C_{0}\left\langle\cdot, \varphi_{0}\right\rangle \varphi_{0}$ où $\varphi_{0} \in H_{-a}^{2}\left(\mathbb{R}^{n}\right)$ vérifie

$$
\Pi_{-} \varphi_{0}=\varphi_{0} \quad \text { et } \quad H \varphi_{0}=0
$$

TOME $124-1996-\mathrm{N}^{\circ} 3$ 
Ainsi, si $\varphi$ est un vecteur propre unitaire associé à $\mu\left(t_{0}, 0,0\right)$, on a :

$$
\partial_{u} \mu\left(t_{0}, 0,0\right)=\left\langle\partial_{u} \Gamma_{+}\left(t_{0}, 0,0\right) \varphi, \varphi\right\rangle=C_{0}\left|\left\langle\varphi_{0}, U A_{-+}\left(t_{0}\right) \varphi\right\rangle\right|^{2} .
$$

Supposons $\left\langle\varphi_{0}, U A_{-+}\left(t_{0}\right) \varphi\right\rangle=0$. Soit

$$
\psi_{+}=a \varphi+b \phi_{+} \in \Pi_{+}\left(\mathcal{A}_{1}\right)
$$

où $\phi_{+}$est un vecteur unitaire orthogonal à $\varphi$ et $|a| \leq 1$ et $|b| \leq 1$ deux constantes; nous fixerons un choix de ces variables plus loin. Soit $P_{+}$le projecteur orthogonal sur $\psi_{+}$. Perturbons $A(t)$ en $A^{\epsilon}(t)=A(t)+\epsilon P_{+}$. Alors $A^{\epsilon}(t)$ vérifie encore l'hypothèse (H.3). On peut étudier $H+A^{\epsilon}(t)$. Comme dans le cas $\epsilon=0$, on lui associe $\Gamma_{+}^{\epsilon}(t, \lambda, u)=\Gamma_{+}(t, \lambda, u)+\epsilon P_{+}$. Donc, pour $\epsilon$ assez petit, $H_{+}+\Gamma_{+}^{\epsilon}(t, \lambda, u)$ admet une unique valeur propre simple voisine de 0 que nous noterons $\mu(\epsilon, t, \lambda, u)$. Le calcul de perturbation classique nous dit que :

$$
\begin{aligned}
& \partial_{t} \mu(\epsilon, t, \lambda, u)_{\mid\left(0, t_{0}, 0,0\right)}=\partial_{t} \mu(t, \lambda, u)_{\mid\left(t_{0}, 0,0\right)}=c \neq 0, \\
& \partial_{\epsilon} \mu(\epsilon, t, \lambda, u)_{\mid\left(0, t_{0}, 0,0\right)}=\left\langle\varphi, \psi_{+}\right\rangle^{2}=a^{2} .
\end{aligned}
$$

Par le théorème d'inversion locale on construit au voisinage de 0 une fonction $\epsilon \mapsto t(\epsilon)$ analytique en $\epsilon$ tel que $t(0)=t_{0}$ et $\mu(\epsilon, t(\epsilon), 0,0)=0$ pour $\epsilon$ assez petit. On a $\partial_{\epsilon} t(0)=-a^{2} / c$.

Soit $\varphi(\epsilon, t, \lambda, u)$ un vecteur unitaire associé à la valeur propre $\mu(\epsilon, t, \lambda, u)$ et analytique dans toutes les variables. On notera $\varphi(\epsilon)=\varphi(\epsilon, t(\epsilon), 0,0)$. Par définition,

$$
\left(H_{+}+\Gamma_{+}(t(\epsilon), 0,0)+\epsilon P_{+}\right) \varphi(\epsilon)=0 \text {. }
$$

Donc, en dérivant en $\epsilon$, pour $\epsilon=0$ on a :

$$
\left(H_{+}+\Gamma_{+}\left(t_{0}, 0,0\right)\right) \partial_{\epsilon} \varphi(0)=-\partial_{\epsilon} t(0) \partial_{t} \Gamma_{+}\left(t_{0}, 0,0\right) \varphi-P_{+} \varphi .
$$

Par construction $\left\langle-\partial_{\epsilon} t(0) \partial_{t} \Gamma_{+}\left(t_{0}, 0,0\right) \varphi-P_{+} \varphi, \varphi\right\rangle=0$, c'est-à-dire que $-\partial_{\epsilon} t(0) \partial_{t} \Gamma_{+}\left(t_{0}, 0,0\right) \varphi-P_{+} \varphi$ est orthogonal au noyau de $H_{+}+\Gamma_{+}\left(t_{0}, 0,0\right)$. On a :

$$
\partial_{\epsilon} \varphi(0)=-\left(H_{+}+\Gamma_{+}\left(t_{0}, 0,0\right)\right)^{-1}\left(\partial_{\epsilon} t(0) \partial_{t} \Gamma_{+}\left(t_{0}, 0,0\right) \varphi-P_{+} \varphi\right) .
$$

Par le calcul fait précédemment, on a :

$$
\partial_{u} \Gamma_{+}^{\epsilon}(t(\epsilon), 0,0)=A_{+-}(t(\epsilon))\left\{U^{*}(t(\epsilon)) G_{-}(0) U(t(\epsilon))\right\} A_{-+}(t(\epsilon))
$$


donc, comme $\left\langle\varphi_{0}, U A_{-+}\left(t_{0}\right) \varphi\right\rangle=0$, pour $\epsilon$ voisin de 0 ,

$$
\begin{aligned}
& \partial_{u} \mu(\epsilon, t(\epsilon), 0,0) \\
& =C_{0} \mid \epsilon\left(\partial_{\epsilon} t\left\langle\partial_{t} A_{+-}\left(t_{0}\right) U^{*} \varphi_{0}, \varphi\right\rangle+\partial_{\epsilon} t\left\langle A_{+-}\left(t_{0}\right) \partial_{t} U^{*} \varphi_{0}, \varphi\right\rangle\right. \\
& \quad-\partial_{\epsilon} t\left\langle A_{+-}\left(t_{0}\right) U^{*}\left(t_{0}\right) \varphi_{0},\left(H_{+}+\Gamma_{+}\left(t_{0}, 0,0\right)\right)^{-1} \partial_{t} \Gamma_{+}^{\epsilon} \varphi\right\rangle \\
& \left.\quad+\left\langle A_{+-}\left(t_{0}\right) U^{*} \varphi_{0},\left(H_{+}+\Gamma_{+}\left(t_{0}, 0,0\right)\right)^{-1} P_{+} \varphi\right\rangle+\mathrm{O}(\epsilon)\right)\left.\right|^{2}
\end{aligned}
$$

Si on choisit maintenant $\phi_{+}=\left(H_{+}+\Gamma_{+}\left(t_{0}, 0,0\right)\right) A_{+-} U^{*} \varphi_{0}$, on obtient pour des constantes $C$ et $C^{\prime}$ indépendantes de $a$ et $b$,

$$
\partial_{u} \mu(\epsilon, t(\epsilon), 0,0)=C \epsilon^{2}\left|C^{\prime} a^{2}+a b+\mathrm{O}(\epsilon)\right|^{2} .
$$

Donc si on choisit $a$ suffisamment petit par rapport à $b$ et $\epsilon$ assez petit, on obtient

$$
\partial_{u} \mu(\epsilon, t, \lambda, u)_{\mid(\epsilon, t(\epsilon), 0,0)} \neq 0 .
$$

Ceci achève la preuve de la généricité de l'hypothèse $a_{0,0,1} \neq 0$ en dimension $n \geq 5$.

Alors, pour répondre à la question de M.Sh. Birman, on peut dire les choses suivantes :

a) pour $n=3$, on a $m=p=1$ et $q=2$; dans ce cas la surface de Riemann a deux feuillets. La valeur propre se transforme en résonance,

b) pour $n$ impair $n>3$, on a $m=q=2$ et $p=n$; dans ce cas la surface de Riemann a deux feuillets. Donc pour $t>t_{0}$ et $t$ proche de $t_{0}$, il y a en plus de la valeur propre une autre résonance qui atteint le seuil en même temps que la valeur propre. Pour $t<t_{0}$ il y a deux résonances près du seuil,

c) en dimension paire $>2$, pour $t>t_{0}$ et $t$ proche de $t_{0}$, en plus de la valeur propre, il y a une résonance sur chaque feuillet de la surface de Riemann. La valeur propre et les résonances arrivent toutes ensemble au seuil et pour $t<t_{0}$, on retrouve une résonance sur chaque feuillet, la valeur propre s'étant transformée en résonance.

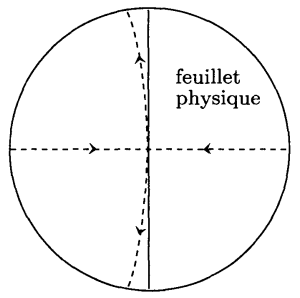

Dans la figure ci-contre, les branches de résonances en dimension impaire supérieure à 3 sont représentées par des courbes en pointillé. Le segment vertical en trait gras se projette sur la coupure, la partie droite sur le feuillet physique et la partie gauche sur l'autre feuillet. Les flèches indiquent le sens de parcours des

TOME $124-1996-\mathrm{N}^{\circ} 3$ 
résonances quand $t$ décroît, ainsi pour $t>t_{0}$ on a les deux branches horizontales et pour $t<t_{0}$ on a les deux courbes «verticales».

\section{BIBLIOGRAPHIE}

[ADH] Alama (S.), Deift (P.A.) and Hempel (R.). - Eigenvalue branches of the Schrödinger operator $H-\lambda W$ in a gap of $\sigma(H)$, Comm. Math. Phys., t. 121, 1989, p. 291-321.

[Ba-Sk] BAlslev (E.) and Skibstedt (E.). - Resonance theory of two-body Schrödinger operators, Ann. Inst. Henri Poincaré, sér. Phys. Théor., t. 51, 1989, p. 129-154.

[Bi] Birman (M.Sh.). - On the discrete spectrum in the gaps for a perturbed periodic second-order operator, Funct. Anal. Appl., t. 25, 4, 1991, p. 158-161.

[CFKS] Cycon (H.L.), Froese (R.G.), Kirsch (W.) and Simon (B.). Schrödinger operators. - Springer, Berlin Heidelberg New York, 1987 .

[Gé1] GÉrARd (C.). - Resonance theory in atom surface scattering, Comm. Math. Phys., t. 126, 1989, p. 263-290.

[Gé2] GÉRARD (C.).- Resonance theory for periodic Schrödinger operators, Bull. Soc. Math. France, t. 118, 1990, p. 27-54.

[Ho] Howland (J.). - The Livsic matrix in perturbation theory, J. Math. Anal. Apppl., t. 50, 1975, p. 415-437.

[Kl] KLOPP (F.). - Resonances for perturbations of a semi-classical periodic Schrödinger operator, to appear in Arkiv. Math..

[Or] ORTH (A.). - Quantum mechanical resonance and limiting absorption : the many-body case, Comm. Math. Phys., t. 126, 1990, p. $559-573$.

[Rai] RaIkov (G.D.). - Eigenvalue asymptotics for the Schrödinger operator with perturbed periodic potential, Invent. Math., t. 110, 1992, p. 75-93.

[ReSi] ReED (M.) and Simon (B.). - Methods of modern mathematical physics, vol. IV : analysis of operators. - Academic Press, New York, 1978.

[Sob] Sobolev (A.V.). - Weyl asymptotics for the discrete spectrum of the perturbed Hill operator, Adv. Sov. Math., Providence RI, t. 7, 1991, p. 159-178. 